Measurability Theorems for Stochastic Extremals.
P. $\operatorname{Kal1}{ }^{*}$
W. Oettli
[42.]

Summary: Measurability of the optimal value is proved for a rather general class of parametric optimization problems. The class considered includes in particular the stochastic convex programs. The measurability of the optimal solutions is discussed for a special case.

* Institut für Operations Research und Mathematische Methoden der Wirtschaftswissenschaften, Universität Zürich, CH-8006 Zürich, Switzerland

** Fakultät für Mathematik und Informatik, Universität Mannheim, D-6800 Mannheim, Germany. 


\section{Measurability Theorems for Stochastic Extremals}

\section{P. Kall (Zürich), W. Oettli (Mannheim)}

In [1] a direct and elementary proof was given for the measurability of the optimal value of a stochastic linear program. It turns out that the same technique yields measurability statements for very general nonlinear optimization problems, too.

1. Let $\Omega$ be some measurable space, and let $X$ be some subset of $\mathbb{R}^{n}$. Throughout we suppose that $X$ contains a countable dense subset $\Xi=\left\{\xi_{i}\right\}_{i \in \mathbb{N}}$. Let the functions $F: X \times \Omega \rightarrow \mathbb{R}$ and $f: X \times \Omega \rightarrow \mathbb{R}$ be measurable on $\Omega$ for every $x \in X$. We are interested in the measurability of the optimal value

$$
\Phi(\omega)=\left\{\begin{array}{l}
\inf _{x}\{F(x, \omega) \mid x \in X, f(x, \omega) \leq 0\} \text { if }\{x \mid x \in X, f(x, \omega) \leq 0\} \neq \emptyset, \\
+\infty \text { e1se. }
\end{array}\right.
$$

Let us define in addition for $n \in \mathbb{N}$

$$
\tau_{n}(\omega)=\left\{\begin{array}{l}
\inf _{x}\left\{F(x, \omega) \mid x \in X, f(x, \omega) \leq \frac{1}{n}\right\} \text { if }\left\{x \mid x \in X, f(x, \omega) \leq \frac{1}{n}\right\} \neq \emptyset, \\
+\infty \text { else, }
\end{array}\right.
$$

and for all $n \in \mathbb{N}$, $i \in \mathbb{N}$

$$
\Phi_{\text {in }}(\omega)=\left\{\begin{array}{l}
F\left(\xi_{i}, \omega\right) \text { if } f\left(\xi_{i}, \omega\right) \leq \frac{1}{n}, \\
+\infty \text { else. }
\end{array}\right.
$$

According to our measurability assumptions $\Phi_{\text {in }}(\omega)$ is an extended real valued measurable function for every $n \in \mathbb{N}$ and $i \in \mathbb{N}$.

Lemma: Let $\mathrm{F}$ and $\mathrm{F}$ be upper semicontinuous on $\mathrm{X}$ for every $\omega \in \Omega$, and suppose that $\sup _{\mathrm{n}} \tau_{\mathrm{n}}(\omega) \geq \Phi(\omega)$ for $a l l \omega \in \Omega$. Then $\Phi(\omega)$ is measurable.

Proof: For all $n \in \mathbb{N}$ and $i \in \mathbb{N}$ we have $\tau_{n}(\omega) \leq \Phi_{\text {in }}(\omega)$, implying $\tau_{n}(\omega) \leq$ $\leq \inf _{i} \Phi_{i n}(\omega)$, and hence $\sup _{n} \tau_{n}(\omega) \leq \sup _{n} i n f_{i} \Phi_{i n}(\omega)$. By hypothesis then

$$
\Phi(\omega) \leq \sup _{n} \inf _{i} \Phi_{i n}(\omega) \text {. }
$$


To show the converse inequality we suppose first that $\mathbf{s}(\omega)<+\infty$. Then there exist points $x \in X$ satisfying $f(x, \omega) \leq 0$, and - due to the upper semicontinuity

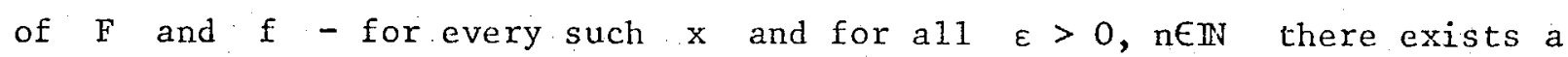
$\xi_{i} \in E$ such that

$$
f\left(\xi_{i}, \omega\right) \leq \frac{1}{n}, F\left(\xi_{i}, \omega\right) \leq F(x, \omega)+\varepsilon_{0}
$$

Hence for every $n \in \mathbb{N}$ we have inf ${ }_{i} \Phi_{i n}(\omega) \leq F(x, \omega)+\varepsilon$, and therefore $\sup _{n} \inf _{i} \Phi_{\text {in }}(\omega) \leq F(x, \omega)+\varepsilon$. Since this inequality is true for all $x \in\{x \mid x \in X, " f(x, \omega) \leq 0\}$ and for every $\varepsilon>0$, we have

$$
\sup _{n} \inf _{i} \Phi_{\text {in }}(\omega) \leq \Phi(\omega) \text {. }
$$

Inequality (2) is trivially satisfied if $\Phi(\omega)=+\infty$. From (1) and (2) we obtain

$$
\Phi(\omega)=\sup _{n} \inf _{i} \Phi_{\text {in }}(\omega)
$$

Since the infimum and supremum of countably many measurable functions is again measurable, the Lemma follows. q.e.d.

The assumption sup ${ }_{n}{ }_{n}(\omega) \geq \Phi(\omega)$. may be replaced by the assumption that the Kuhn-Tucker condition holds for all $\omega$ with $\Phi(\omega)<+\infty$. More precisely we have

Theorem 1: Let $F$ and $\mathrm{f}$ be upper semicontinuous on $\mathrm{X}$ for every $\omega \in \Omega$. Suppose that for every $\omega \in\{\omega \mid \Phi(\omega)<+\infty\}$ there exists a real number $u(\omega) \geq 0$ such that

$$
\Phi(\omega) \leq F(x, \omega)+u(\omega) \cdot f(x, \omega) \quad \forall x \in X \quad(K .-T . \text { condition })
$$

and suppose that for every $\omega \in\{\omega \mid \Phi(\omega)=+\infty\}$ we have $\sup _{\mathbf{n}} \tau_{\mathbf{n}}(\omega)=+\infty$. Then $\Phi(\omega)$ is measurable.

Proof: We have to show that $\sup _{n} \tau_{n}(\omega) \geq \dot{\Phi}(\omega)$ for all $\omega$ satisfying $\Phi(\omega)<+\infty$. Then the result follows from the Lemma. According to the Kuhn-Tucker condition assumed, $F(x, \omega) \geq \Phi(\omega)-u(\omega) \cdot \frac{1}{n}$ for all $x \in x$ such that $f(x, \omega) \leq \frac{1}{n}$. Hence $\tau_{n}(\omega) \geq \Phi(\omega)-u(\omega) \cdot \frac{1}{n}$, which implies

$$
\sup _{n} \tau_{n}(\omega) \geq \Phi(\omega)
$$


Corollary 1: If $\mathrm{x}=\mathbb{R}^{\mathrm{n}}$, if $\mathrm{F}$ is convex in $\mathrm{x}$ for every wes, and if $f(x, \omega)=\max _{1 \leq j \leq m} l_{j}(x, \omega)$, where the functions $\ell_{j}$ are linear-affine in $x$, then $\Phi(\omega)$ is measurabie.

Proof: $F$ and $f$ are continuous in $x$, since they are convex over all of $\mathbb{R}^{\mathrm{n}}$. The Kuhn-Tucker condition is satisfied, since it always holds for convex programs with only linear constraints. If the linear system $\ell_{j}(x, \omega) \leq 0$ (with $j=1, \ldots, m$ ) has no solution, then it is a standard result of linear programming that the system $\ell_{j}(x, \omega) \leq \frac{1}{n}(j=1, \ldots, m)$ also has no solution for all sufficiently large $n \in \mathbb{N}$. Thus $\Phi(\omega)=+\infty$ implies $\sup _{n} \tau_{n}(\omega)=+\infty$. The assumptions of Theorem 1 are therefore satisfied. q.e.d.

Corollary 1 implies in particular that the optimal value of a stochastic linear program is measurable.

2. The assumption, made in Theorem 1, that the Kuhn-Tucker condition be satisfied for all $\omega$ with $\Phi(\omega)<+\infty$ is very restrictive, since even for convex programs the Kuhn-Tucker condition generally holds only if $\inf _{x \in X} f(x, \omega)<0$. It is for this reason that we introduce a modified optimal value, $\Psi(\omega)$, defined as

$$
\Psi(\omega)= \begin{cases}\inf _{x}\{F(x, \omega) \mid x \in X, f(x, \omega) \leq 0\} & \text { if } \text { inf }_{x \in X} f(x, \omega)<0, \\ \sup _{n} \tau_{n}(\omega) & \text { if inf } x \in X(x, \omega)=0 \\ +\infty & \text { if inf }{ }_{x \in X} f(x, \omega)>0\end{cases}
$$

Theorem 2: Let $\mathrm{F}$ and $\mathrm{f}$ be upper semicontinuous on X for every wES. Suppose that for all $\omega \in\{\omega \mid$ inf $\mathrm{f}(\mathrm{x}, \omega)<0\}$ there exists a real number $\mathrm{u}(\omega) \geq 0$ such that

$$
\Psi(\omega) \leq \mathrm{F}(\mathrm{x}, \omega)+\mathrm{u}(\omega) \cdot \mathrm{f}(\mathrm{x}, \omega) \quad \forall \mathrm{x} \in \mathrm{X} \quad\left(K_{0}-T \cdot\right. \text { condition). }
$$

Then $\Psi(\omega)$ is measurable.

Proof: As in the proof of the Lemma we have for all $w \in \Omega$ that

$$
\sup _{n} \tau_{n}(\omega) \leq \sup _{n} \inf _{i} \Phi_{i n}(\omega)
$$


If $\inf _{X} f(x, w)<0$ we conclude from the Kuhn-Tucker condition, as in the proof of Theorem 1, that

$$
\Psi(\omega) \leq \sup _{n} \tau_{n}(\omega)
$$

This is also true if inf $f(x, \omega)=0$, from the definition of $\Psi$. If inf $f(x, w)>0$, then there is a real number $\rho(\omega)>0$ such that $f(x, \omega) \geq \rho(\omega)$ for all $x \in X$, implying $\tau_{n}(\omega)=+\infty$ for all $n>\frac{1}{\rho(\omega)}$, and thereby $\Psi(\omega)=\sup _{n} \tau_{n}(\omega)=+\infty$. Hence we have for all $\omega \in \Omega$

$$
\Psi(\omega) \leq \sup _{n} \inf _{i} \Phi_{i n}(\omega) .
$$

On the other hand for all $w$ satisfying inf $f(x, w) \neq 0$ the relation

$$
\sup _{n} \text { inf }_{i} \Phi_{\text {in }}(\omega) \leq \Psi(\omega)
$$

follows from the upper semicontinuity of $F$ and $f$, as in the proof of the Lemma. Let now inf $f(x, w)=0$. Choose $n \in \mathbb{N}$ and $\varepsilon>0$ arbitrarily. Then for every $x \in X$ satisfying $f(x, w) \leq \frac{1}{2 n}$ there exists, according to the upper semicontinuity of $F$ and $€$, an element $\xi_{i} \in E$ such that

$$
f\left(\xi_{i}, \omega\right) \leq f(x, \omega)+\frac{1}{2 n} \leq \frac{1}{n}, F\left(\xi_{i}, \omega\right) \leq F(x, \omega)+\varepsilon
$$

Hence $\Phi_{\text {in }}(\omega) \leq F(x, \omega)+\varepsilon$ and $\inf _{i} \phi_{\text {in }}(\omega) \leq \tau_{2 n}+\varepsilon$. Since $\varepsilon$ was arbitrary we get

$$
\sup _{n} \inf _{i} \Phi_{\text {in }}(\omega) \leq \sup _{n} \tau_{2 n}(\omega) \leq \sup _{n} \tau_{n}(\omega)
$$

Since $\sup _{n} \tau_{n}(\omega)=\Psi(\omega)$ in the case under consideration, (5) again holds. In conclusion we have from (4), (5)

$$
\Psi(\omega)=\sup _{n} \inf _{i} \Phi_{\text {in }}(\omega),
$$

which proves the measurability of $\Psi(\omega)$. q.e.d.

Corollary 2: Let $\mathrm{X}$, be a convex set, and let $\mathrm{F}$ and $\mathrm{f}$ be convex functions in $x$ for every $\omega \in \Omega$. Then $\Psi(\omega)$ is measurable.

Proof: The Kuhn-Tucker condition, as required in Theorem 2, is satisfied, since $\inf _{X} f(x, \omega)<0$ is the well-known Slater-condition, the latter implying in the convex case the validity of the Kuhn-Tucker condition. The requirement of upper semi- 
continuity may be dropped in the convex case. Indeed, the upper semicontinuity of $F$ (resp. f) was used only to conclude that for every $x \in X$ and $\varepsilon>0$ there exists $\xi_{i} \in \Xi$ satisfying

$$
F\left(\xi_{i}, \omega\right) \leq F(x, \omega)+\varepsilon \text {. }
$$

In the convex case the same conclusion may be reached as follows: Let $z$ be an arbitrary point in the relative interior of $x$. Then $x_{\lambda}=x+\lambda(z-x)$ with $0<\lambda \leq 1$ is also in the relative interior of: $X$. Since $F$ is convex in $x$ we have

$$
F\left(x_{\lambda}, \omega\right) \leq F(x, \omega)+\lambda(F(z, \omega)-F(x, \omega)) .
$$

Choose $\lambda>0$ so small that

$$
F\left(x_{\lambda}, w\right) \leq F(x, \omega)+\frac{\varepsilon}{2} .
$$

Since $x_{\lambda}$ is in the relative interior of $X$, since $F$ - as a convex function - is continuous in the relative interior of its domain $x$, and since $\Xi$ is dense in $x$, there exists: $\xi_{i} \in \Xi$ such that

$$
\left|F\left(\xi_{i}, \omega\right)-F\left(x_{\lambda}, \omega\right)\right| \leq \frac{\varepsilon}{2} .
$$

From (7) and (8) follows (6). $q . e . d$.

3. We would like to point out that Corollary 1 could also be derived from Theorem 2 instead of from Theorem 1, since it may be shown under the assumptions of Corollary 1 that $\Phi$ and $\Psi$ coincide. Under the weaker assumptions of Corollary 2 , however, $\Phi$ and $\Psi$ may differ, as the following examples show: Choose

$$
x=\left\{\left(x_{1}, x_{2}\right) \mid x_{1} \geq 1\right\} \subset \mathbb{R}^{2}, F\left(x_{1}, x_{2}, w\right)=x_{2}, f\left(x_{1}, x_{2}, w\right)=\left(x_{2}\right) 2 / x_{1} .
$$

Then $\Phi(\omega)=0$, but $\Psi(\omega)=\sup _{n} \tau_{n}(\omega)=-\infty$. To take another example, let

$$
X=[0,1] \subset \mathbb{R}^{1}, F(x, \omega) \equiv 0, f(0, \omega)=1, f(x, \omega)=x^{2} \text { for } x>0 .
$$

Then $\Phi(\omega)=+\infty$, but $\Psi(\omega)=0$. A further comparison of $\Phi$ and $\Psi$ therefore seems appropriate.

Theorem 3: If $\mathrm{x}$ is compact, and $\mathrm{F}$ and $\mathrm{f}$ are Iower semicontinuous in $\mathrm{x}$ for every $\omega \in \Omega$, then $\Phi(\omega)=\Psi(\omega)$. 
Proof: We have to show that if $\inf _{X} f(x, \omega)=0$ then $\sup _{n} \tau_{n}(\omega)=\Phi(\omega)$. Obviously $\sup _{n} \tau_{n}(\omega) \leq \Phi(\omega)$. On the other hand, by lover semicontinuity and compactness, for every $n \in \mathbb{N}$ there exists $x_{n} \in X$ satisfying

$$
f\left(x_{n}, \omega\right) \leq \frac{1}{n}, F\left(x_{n}, \omega\right)=\tau_{n}(\omega)
$$

Let $\left\{\mathrm{x}_{\mathrm{j}}\right\}$ be a subsequence converging to some $\mathrm{x}_{\mathrm{o}} \in \mathrm{x}_{0}$. Then, by lower semicontinuity and by the monotonicity of $\tau_{n}(\omega)$,

$$
f\left(x_{0}, \omega\right) \leq \underset{j \rightarrow \infty}{\lim \inf } f\left(x_{n_{j}}, \omega\right) \leq 0, F\left(x_{0}, \omega\right) \leq \underset{j \rightarrow \infty}{\lim \inf } F\left(x_{n_{j}}, \omega\right) \leq \sup _{n} \tau_{n}(\omega),
$$

implying $\sup _{n} \tau_{n}(\omega) \geq \Phi(\omega)$.

q.e.d.

Combining Theorems 2 and 3 one can derive measurability statements for $\Phi(\omega)$. In particular we obtain very easily the following result which is contained in $[2$, Corollary 4.3].

Corollary 3: Let $\mathrm{x}$ be a closed convex set, and tet $\mathrm{F}$ and $\mathrm{f}$ be lower. semicontinuous convex functions on $\mathrm{X}$ for every wES. Then $\Phi(\omega)$ is measurable.

Proof: For all $k \in \mathbb{N}$, denote by $\Phi_{k}(\omega)$ [resp. $\left.\Psi_{k}(w)\right]$ the functions which are obtained if in the definition of $\Phi[$ resp. $\Psi]$ we replace $X$ : by the compact subset $\mathrm{X}_{\mathrm{k}} \equiv\{\mathrm{x} \mid \mathrm{x} \in \mathrm{X},\|\mathrm{x}\| \leq \mathrm{k}\}$. By Corollary $2 \Psi_{\mathrm{k}}(\omega)$ is measurable. By theorem 3 $\Phi_{k}$ equals $\Psi_{k}$, hence is niseasurable. The measurability of $\Phi$ follows since obviously

$$
\Phi(\omega)=\inf _{k} \Phi_{k}(\omega) \text {. }
$$$$
q \cdot e \cdot d_{s}
$$

4. In this section we want to discuss briefly the measurability of the solution mapping for the case of set-valued constraints.

Let $\mathrm{X}$ be a nonvoid, compact, convex subset of $\mathbb{R}^{\mathrm{n}}$. Let $\Gamma$ be the family of. all nonvoid, closed, convex subsets of $X$. For arbitrary $c \in \Gamma$ define $U(C, \varepsilon)=$ $=\{x \in X \mid \exists \xi \in C:\|x-\xi\| \leq \varepsilon\}$. Obviously $U(C, \varepsilon)$ is also in $\Gamma$. We make $\Gamma$ a metric space by introducing the Hausdorff-distance

$$
\mathrm{d}\left(\mathrm{C}_{1}, \mathrm{C}_{2}\right)=\min \left\{\hat{\theta} \mid \theta \in \mathbb{R}, \mathrm{C}_{1} \subset \mathrm{U}\left(\mathrm{C}_{2}, \theta\right), \mathrm{C}_{2} \subset \mathrm{U}\left(\mathrm{C}_{1}, \theta\right)\right\} \text {. }
$$


Let $\Omega$ be a measurable space. Let $c: \Omega \exists \omega \rightarrow c(\omega) \in \Gamma$ be a measurable mapping, and let $\mathrm{F}: \mathrm{X} \rightarrow \mathbb{R}$ be a continuous, convex function. The function $\Phi(\omega): \Omega \rightarrow \mathbb{R}$ is defined as

$$
\Phi(\omega)=\min \left\{F(x) \mid x E_{c}(\omega)\right\}
$$

Under the assumptions made we may associate with each $w \in \Omega$ a set $\hat{c}(\omega) \in \Gamma-$ the solution set - according to

$$
\hat{c}(\omega)=\{x \in c(\omega) \mid F(x)=\Phi(\omega)\} .
$$

The measurability of $\hat{c}(\omega)$, considered as a multivalued mapping from $\Omega$ into. $\mathbb{R}^{\mathrm{n}}$, has been discussed in [2]. We do not know about practical conditions which ensure the measurability of $\hat{c}(\omega)$ if considered as a singlevalued mapping from $\Omega$ into $\Gamma$. But instead we can show measurability of a multivalued mapping $\gamma$ closely related to $\hat{c}$. Recall that a multivalued mapping

$$
\gamma: \Omega \ni \omega \rightarrow \gamma(\omega) \subset \Gamma
$$

is called measurable if the set

$$
\gamma^{-1}(H) \equiv\{\omega \in \Omega \mid \gamma(\omega) \cap H \neq \phi\}
$$

is measurable in $\Omega$ for any closed subset $H \subset \Gamma$. Now take: as $\gamma$ the multivalued mapping which assigns to each $\omega \in \Omega$ the family of all nonvoid, closed, convex subsets of $\hat{c}(\omega)$. Then we have

Theorem 4: The multivalued mapping $\gamma(\omega)$ is measurable.

Proof: It is easy to see that for arbitraxy $C \in P$ the requirement $c E_{Y}(\omega)$ is equivalent with the two requirements

$$
C \subset c(\omega), \min \{F(x) \mid x \in c(\omega)\} \geq \max \{F(x) \mid x \in C\} \text {. }
$$

The function $F(x)$ is uniformly continuous on the conpact set $X$. This implies that the two functions

$$
m(C)=\min \{F(x) \mid x \in C\}, M(C)=\max \{F(x) \mid x \in C\}
$$

are continuous on $\Gamma$. Now let $H \subset \Gamma$ be closed in $\Gamma$. We have to show that 
$\gamma^{-1}(\mathrm{H})$ is measurable in $\Omega$. Because of (9) we have

$$
\gamma^{-1}(H)=\{\omega \in \Omega \mid \exists C E H: C \subset c(\omega) \& m(c(\omega)) \geq M(C)\}
$$

i.e., $\gamma^{-1}(\mathrm{H})$ is the inverse image with regard to the measurable mapping c(w) of the set

$$
\mathrm{K}=\{\mathrm{D} \in \Gamma \mid \exists C \in \mathrm{H}: \mathrm{C} \subset \mathrm{D} \& \mathrm{~m}(\mathrm{D}) \geq M(C)\}
$$

We show that $K$ is closed in $\Gamma$. Let $\left\{D_{n}\right\}_{n \in \mathbb{N}}$ be a sequence from $K$, converging to some element $D \in \Gamma$. By the definition of $K$ there exists for all $n \in \mathbb{N}$ a set $\mathrm{C}_{\mathrm{n}} \in \mathrm{H}$ such that

$$
\mathrm{C}_{\mathrm{n}} \subset \mathrm{D}_{\mathrm{n}} \& \mathrm{~m}\left(\mathrm{D}_{\mathrm{n}}\right) \geq \mathrm{M}\left(\mathrm{C}_{\mathrm{n}}\right)
$$

In view of our convexity assumptions Blaschke's selection theorem [3] ensures that $\Gamma$ is sequentially compact. Hence $\mathrm{H}$ is also sequentially compact, and there exists a convergent subsequence $C_{n_{j}} \rightarrow C E H$. The continuity of $m$ and $M$ implies in the Iimit

$$
m(D) \geq M(C)
$$

From $C \subset U\left(C_{n}, d\left(C_{n}, C\right)\right), C_{n} \subset D_{n}, D_{n} \subset U\left(D, d\left(D, D_{n}\right)\right)$ it follows that

$$
C \subset U\left(D, d\left(C_{n}, C\right)+d\left(D_{n}, D\right)\right) \text {. }
$$

Since $d\left(C_{n_{j}}, C\right) \rightarrow 0$ and $d\left(D_{n}, D\right) \rightarrow 0$ we have in the limit

$$
\mathrm{C} \subset \mathrm{D} \text {. }
$$

Therefore $D E K$, and $K$ is closed. Since $\gamma^{-1}(H)=c^{-1}(K)$ with $c$ measurable and $\mathrm{K}$ closed, the measurability of the set $\gamma^{-1}(\mathrm{H})$ in $\Omega$ follows. q.e.d.

Note that the function $\Phi(\omega)$ is measurable, being the composition of the measurable mapping $c(\omega)$ and the continuous function $m(c)$.

\section{References}

[1] P. Ka11: Some Remarks on the Distribution Problem of Stochastic Linear Programming. Methods of Operations Research 16 (1973), 189-196. 
[2] R. T. Rockafellar: Measurable Dependence of Convex Sets and Functions on Parameters. J. Math. Anal. Appl. 28 (1969), 4-25.

[3] H. G. Eggleston: Convexity. Cambridge University Press, Cambridge, 1969. 